\author{
LIVIO MELINA \\ Pontificio Istituto Giovanni Paolo II \\ per le scienze del Matrimonio e della Famiglia \\ Ponitificia Università Lateranense, Roma
}

\title{
Interpretazioni di Gaudium et spes e recezione di Humanae vitae
}

La questione del rapporto tra la costituzione pastorale del Concilio Vaticano II Gaudium et spes e l'enciclica del Beato Paolo VI Humanae vitae è ritornata di grande attualità dopo la pubblicazione dell'esortazione post-sinodale Amoris laetitia e in relazione al $50^{\circ}$ anniversario dell'enciclica montiniana, che stiamo celebrando ${ }^{1}$. Basta vedere come ne viene reinterpretato il nesso nel recente volume dei teologi tedeschi Stephan Goertz e Caroline Witting (Goertz, Witting [Hrsg.] 2016, 13-79).

La strategia ermeneutica dei progressisti è però oggi molto diversa da quella seguita cinquant'anni fa: allora si contrapponeva il personalismo di Gaudium et spes al presunto naturalismo di Humanae vitae, sottintendendo che quest'ultima sarebbe stata un passo indietro rispetto al Vaticano II. Ora invece si cerca di contrapporre tra loro due diverse letture possibili della stessa Humanae vitae: quella proposta sulla scorta di Amoris laetitia come un vero e proprio "cambio di paradigma" (Paradigmwechsel) ${ }^{2}$ e quella che sarebbe stata imposta con i pontificati di San Giovanni Paolo II e di Benedetto XVI. La prima, innovando rispetto alla tradizione morale scolastica, ma pretendendo di essere in piena fedeltà con lo spirito del Concilio, adotterebbe lo schema pastorale e flessibile del discernimento "caso per caso", mentre la seconda farebbe ricorso ad un'impostazione rigida, legalistica e dottrinaria. Così il bersaglio polemico per l'interpretazione autentica di Gaudium et spes non è più l'en-

\footnotetext{
${ }^{1}$ Per l'impostazione del problema nella discussione dopo Humanae vitae: Delhaye 1968, 351 -368; Fernández Benito 1994. Del nesso tra i due documenti si era occupato anche Giovanni Paolo II nella Catechesi dell'Udienza generale del 1 agosto 1984.

${ }^{2}$ Kasper 2016, 723-732. Nello stesso senso si muove anche il più articolato intervento di Schockenhoff 2017, 147-158.
} 
ciclica di Paolo VI, ma diventa piuttosto il magistero dei suoi due successori e in particolare l'enciclica Veritatis splendor e la stessa teologia del corpo delle Catechesi del mercoledì del grande papa polacco.

Gaudium et spes, riletta nella luce di Amoris laetitia (o meglio ancora: di certe ermeneutiche progressiste di questa esortazione di papa Francesco) diventerebbe la chiave autenticamente conciliare per poter finalmente fare pace anche con Humanae vitae, smussandone le pretese normative eccessive.

Per entrare nel vivo del conflitto delle interpretazioni con qualche frutto sostanziale, occorre però uscire dagli schematismi piuttosto formali delle contrapposizioni ed affrontare i contenuti dei dibattiti dottrinali e teologici, che mi pare si possano sintetizzare intorno ai seguenti quattro temi principali. In primo piano sta la questione dell'antropologia, in riferimento al rapporto tra persona e natura e quindi alla legge naturale; ci chiederemo perciò: quale personalismo? In secondo luogo, dovremo saggiare il rapporto tra Gaudium et spes ed Humanae vitae sulla questione della gerarchia dei fini del matrimonio, domandandoci: quale amore? In una terza tappa entreremo nel dibattito specificamente morale del rapporto tra norme morali e coscienza e ci chiederemo: quale coscienza? Infine verrà il rapporto tra dottrina e pastorale; e la domanda sarà: quale pastorale?

\section{Quale personalismo?}

“Quale personalismo?”, dunque. È questa la prima fondamentale questione su cui saggiare la qualità delle interpretazioni di Gaudium et spes in rapporto alla recezione di Humanae vitae. Infatti un'etica presuppone sempre un'antropologia, benché ne sia epistemologicamente autonoma ${ }^{3}$. La verità sull'uomo e sul suo bene è a fondamento di tutti i principi della morale. Per questo, la visione della persona umana e dell'amore, che la costituzione pastorale del Vaticano II delinea, dovrebbero essere anche alla base della dottrina etica insegnata dall'enciclica Humanae vitae ${ }^{4}$.

Gaudium et spes è un testo profondamente personalistico, che ha inteso illustrare la dignità singolare dell'uomo e la sua integrale vocazione nei termini di una chiamata alla communio personarum (n. 12), che si realizza mediante il dono sincero di sé (n. 24). Questa costitutiva apertura della persona alle relazioni si basa teologicamente sul fatto che l'essere umano, unica creatura voluta per se stessa, è stato creato "ad immagine e somiglianza di Dio" e che questa

\footnotetext{
${ }^{3}$ Sulla questione dell'originalità della conoscenza morale si vedano: Melina 1987, Pérez-Soba 2011.

${ }^{4}$ In merito si veda il saggio di Wojtyła 1978, 125-145.
} 
dimensione rispecchia in certo modo il mistero trinitario divino. Ora è proprio nel matrimonio, che tale vocazione al dono e alla comunione si realizza in una modalità vocazionale specifica, configurando un cammino di santificazione (nn. 48 e 49) ${ }^{5}$. Possiamo così cogliere il passo in avanti che la costituzione pastorale del Vaticano II rappresentò per la comprensione del sacramento del matrimonio nell'orizzonte dell'amore: il suo aggancio all'esperienza e il suo fondamento in Cristo, che rivela l'amore del Padre e apre una partecipazione al suo legame sponsale con la Chiesa (Cf. Granados 2014, 33-59).

Humanae vitae, da parte sua, fa esplicito riferimento al fatto che «gli sposi per mezzo della reciproca donazione personale, loro propria ed esclusiva, tendono alla comunione dei loro esseri» (n. 8). Infatti per cogliere la vera natura e nobiltà dell'amore coniugale bisogna considerarlo nella sua sorgente suprema: Dio, che è Amore. Così, pur utilizzando meno il linguaggio della "persona" e più quello dell'"essere umano" ", l'enciclica montiniana si richiama all'antropologia personalistica di Gaudium et spes.

Va tuttavia osservato che la questione del personalismo conciliare non è semplice e senza difficoltà ermeneutiche. Tale problematicità è colta in modo acuto da Karol Wojtyla in un'allocuzione svolta a conclusione di un Convegno di teologi polacchi del 1973, di cui riportiamo un passaggio altamente significativo della sua interpretazione globale del Vaticano II:

è sufficiente leggere attentamente non solo Gaudium et spes, ma anche un altro documento del Concilio, la Costituzione Dei Verbum. Quest'ultima tratta specificamente della concezione personalista della Rivelazione, e quindi della conseguente concezione personalista della teologia. Per quanto riguarda la teologia, siamo forse i testimoni di un cambiamento di prospettiva, che qualificherei come il passaggio da un paradigma più "cosmologico" a una prospettiva più "personalista". Preferisco definirlo così, piuttosto che dire che si è verificato un mutamento da una teologia della natura ad una teologia della persona, che sarebbe a mio avviso, utilizzare un linguaggio ambiguo e ingiustificato. La teologia è sempre stata una teologia della persona e dunque anche una teologia della natura (K. Wojtyla, "Słowo końcowe" [Allocuzione conclusiva], in Wojtyła [1973-1974], 243-263).

Purtroppo è proprio questo "linguaggio ambiguo e ingiustificato", che, contrapponendo persona a natura, ha dato luogo ad ermeneutiche discutibili di Gaudium et spes. Già la corrente dominante nel personalismo filosofico aveva

${ }^{5} \mathrm{Cf}$. «I coniugi mutuamente si danno e si ricevono» (GS 48); «Un tale amore, unendo assieme valori umani e divini, conduce gli sposi al libero e mutuo dono di se stessi» (GS 49).

${ }^{6}$ L'osservazione è di Wojtyla 1978, 131, che afferma: «l'enciclica HV è un documento piuttosto 'umanistico' che 'personalistico'. Nello stesso tempo è difficile intendere questo umanesimo altrimenti che in modo personalistico». 
mostrato alcune gravi carenze 7 . Pur nell'intento encomiabile di "difendere la persona" dalla sua omologazione in un mondo di cose e dall'oppressione dei totalitarismi ideologici, taluni aspetti antropologici erano stati trascurati. Segnalo tre punti critici principali: (1) l'interpersonalità era vista talvolta solo come intersoggettività, in cui il riferimento diventa la coscienza o le coscienze (cf. E. Husserl, M. Nédoncelle), smarrendo la concretezza storica e la relazionalità sociale degli esseri umani; (2) la dimensione etica veniva trattata in modo sbrigativo aprendo la strada ad un certo relativismo antinormativista, non lontano dall"'etica di situazione" (B. Häring) ${ }^{8}$; (3) infine la tentazione di fare dell'uomo un fine a se stesso, in una svolta antropocentrica (cf. I. Kant, K. Rahner), che lo chiude nell'orizzonte immanentistico dell'autorealizzazione.

Il punto cruciale del discrimine tra le antropologie e le teologie che si ispirano al personalismo era, come aveva ben visto Wojtyla, proprio la visione della natura in relazione alla persona. L'adozione di un metodo dialettico, che contrappone i due termini di persona e natura, ha condotto ad una serie di falsi dualismi e di aporie, che destrutturano l'etica, come quelle tra libertà e verità, tra coscienza e legge, tra opzione fondamentale e atto, delle quali si è occupato il secondo capitolo dell'enciclica Veritatis splendor. Il dualismo spirito-materia ha un influsso molto forte sul modo in cui l'uomo guarda a se stesso. Il suo corpo viene ridotto a strumento da usare da parte di una libertà che progetta e decide e che può manipolarlo senza limiti. Esso è visto come una cosa, distinta dal soggetto, sulla quale sarebbero le scienze positive a determinare le regole di funzionamento, senza che vi siano valori morali da rispettare. Così alla dimensione spirituale della persona è assegnata una libertà senza fini, che permetterebbe di determinare soggettivamente i contenuti delle azioni, senza riferimento a verità che la precedano e che possano orientarla e vincolarla normativamente? .

Un tale personalismo spiritualistico ha naturalmente come sua conseguenza la messa in questione, o per lo meno la radicale destrutturazione del concetto teologico di legge naturale, che l'enciclica Humanae vitae pone alla base della sua argomentazione (n. 4). Le insufficienze del modello razionalista moderno, che la presenta come uno schema di precetti, deduttivo a priori da una natura umana immutabile e astorica ${ }^{10}$, diventano il pretesto per un rifiuto puro e semplice o per un suo svuotamento formalistico, nello schema trascendentale di taluni moralisti tedeschi, per i quali essa non sarebbe che l'imperativo

${ }^{7}$ Si veda: Pérez-Soba, Gałuszka (a cura di) 2013, e in particolare: Pérez-Soba 2013, 33-78. Una presentazione generale ed una antologia si trova in Rigobello 1975.

${ }^{8}$ Si veda ad esempio l'opera di Häring 1971.

${ }^{9}$ È la tesi del significato solo "pre-morale" dei beni umani, proposta da Janssens 1972, 115-156.

${ }^{10}$ Per una revisione critica di questo modello: Maritain 1985. In merito si è pronunciatala Commissione Teologica Internazionale 2009, n. 33. 
di trovare la soluzione più ragionevole nelle circostanze specifiche dell'agire concreto. Altrimenti si cadrebbe in una funesta "fallacia naturalistica", che finisce col sacralizzare indebitamente la fisiologia della sessualità e della procreazione ${ }^{11}$.

A questa deriva del soggettivismo trascendentale oppone resistenza un testo del n. 51 di Gaudium et spes, che richiamando i criteri di moralità degli atti propri dei coniugi, così afferma: «Il carattere morale dell'agire non dipende solo dalla sincerità dell'intenzione e dalla valutazione dei motivi, ma va determinato sulla base di criteri oggettivi, desunti dalla natura della persona e dei suoi atti» ("ex personae eiusdemque actuum natura desumptis, determinari debet"). Ma ciò che non è possibile piegare a interpretazioni interessate, lo si può semplicemente cambiare. E così in molte traduzioni in lingua volgare moderna, tra cui quelle italiana e tedesca, il termine "natura" è stato sostituito con "dignità". Chi non controlla il latino del testo ufficiale, subisce quindi una distorsione. Addirittura il noto moralista Bernhard Häring arriva a presentare questo come prova del superamento del criterio della natura in nome del personalismo: un personalismo senza la natura o addirittura contro la natura $^{12}$. La manipolazione è tanto più grave in quanto la storia della redazione (Redaktionsgeschichte) mostra che l'inserimento del riferimento alla "natura della persona e dei suoi atti" invece che alla "dignità", era stato esplicitamente richiesto da Papa Paolo VI, in uno dei quattro modi proposti il 23 novembre 1965, ed accolto dalla Commissione per il testo poi approvato in aula ${ }^{13}$.

L'enciclica Humanae vitae, invece di eliminare il riferimento oggettivo al criterio della natura, lo integrerà nella prospettiva della cooperazione del soggetto, mediante il linguaggio dei "significati" dell'atto coniugale (n.12), che collegano ciò che tale atto "oggettivamente" significa col significato che gli sposi gli conferiscono nella dimensione cosciente e soggettiva della loro cooperazione con il disegno di Dio Creatore. Ciò, sottolinea Karol Wojtyła in un suo studio del 1978, «rappresenta un certo progresso (di Humanae vitae) rispetto alla costituzione Gaudium et spes 51» (Wojtyla 1978, 136). L'osservazione è preziosa per definire la strada dell'autentico personalismo, in grado di collegare l'antropologia di Gaudium et spes con le indicazioni etiche dell' $\mathrm{Hu}$ manae vitae, in una duplice direzione.

In primo luogo essa apre alla considerazione della soggettività morale del corpo. È una pista già suggerita nel cosiddetto "Memoriale di Cracovia", cioè in quel documento che l'Arcivescovo Wojtyła, impossibilitato a partecipare alle riunioni della Commissione pontificia per il divieto delle autorità comuni-

${ }^{11}$ È la tesi di Fuchs 1996.

${ }^{12}$ Cf. Häring 1971; nello stesso senso, sorprendentemente si muoveva anche la interpretazione del giovane teologo Ratzinger 1966, 235-239.

${ }^{13}$ Cf. Gil Hellin 1998, 359-372. Un'utile sinossi si trova in Marengo 2014, 102-104. 
ste, inviò nel 1968 a Paolo VI, come contributo alla discussione di un gruppo di teologi polacchi ${ }^{14}$. La tensione tra persona e natura, presente nel testo di Gaudium et spes e risolta dualisticamente nelle interpretazioni "progressiste", viene integrata in una comprensione del corpo umano non come aspetto separato dalla persona, da dominare, ma come "corpo proprio", che partecipa in pienezza al carattere personale dell'uomo (Cf. Granados 2013, 81-86), facendo parte della sua identità. La stessa costituzione conciliare aveva indicato la linea di una visione unitaria dell'uomo, "corpore et anima unus" (n. 14), ma non l'aveva sviluppata e collegata al tema della natura. Si tratta della tematica della "teologia del corpo", che San Giovanni Paolo II tratterà nelle Catechesi del mercoledì, che sono volte ad illustrare i fondamenti antropologici di $\mathrm{Hu}$ manae vitae. L'enciclica Veritatis splendor completerà poi questa rinnovata comprensione personalistica e dinamica della legge naturale, parlando di "soggettività morale del corpo" (n. 48; In merito: Melina, Pérez-Soba [a cura di] 2012) e offrendo spunti per una rilettura delle inclinazioni naturali nella prospettiva dell'amore (nn. 50-53).

In secondo luogo, l'affermazione di Wojtyła radica teologicamente la natura nel disegno di Dio Creatore e Redentore: creato come imago Dei, l'uomo trova luce definitiva al suo mistero solo nel mistero del Verbo incarnato (GS 22). E qui la sua natura cessa di essere un mero "fatto" estrinseco alla libertà, e può venire compresa come un dono di Dio, nell'orizzonte della sua vocazione alla comunione. E' proprio questo l'appello di Humanae vitae, che al n. 7 chiede di considerare il problema della natalità «nella luce di una visione integrale dell'uomo e della sua vocazione, non solo naturale e terrena, ma anche soprannaturale ed eterna».

\section{Quale amore?}

Un secondo punto critico per verificare la coerenza di Gaudium et spes con Humanae vitae, è rappresentato dalla dottrina circa la gerarchia dei fini del matrimonio, che era stata oggetto di intenso dibattito negli anni precedenti il Vaticano II (Sul tema: Mattheeuws 1989; Mazzocato 2006, 249-275; Granados 2013, 53-56). La tesi classica, di matrice agostiniana e tomista (Sant'Agostino, De bono coniugali; San Tommaso d'Aquino, Summa Theologiae, Supplementum, q. 67), nel distinguere i tre beni: proles, fides e sacramentum, aveva identificato come fine primario la procreazione, in quanto esso si colloca ad

${ }^{14}$ Cf. "Memoriale di Cracovia. I fondamenti della dottrina della Chiesa riguardo ai principi della vita coniugale", in Gałuszka 2017, 473-516. Lo studio di Gałuszka è essenziale per cogliere l'importanza decisiva e la novità dell'apporto di Wojtyła alla preparazione e alla recezione dell'enciclica montianiana, e pubblica anche fonti inedite. 
un livello profondo dell'essere umano, comune anche ad altre specie animali, mentre l'aiuto reciproco e fedele, pur essenziale e per se sufficiente, restava in posizione subordinata. Il riconoscimento del primato della persona sembrava chiedere un ribaltamento di questa gerarchia, ponendo in primo piano l'amore dei coniugi. Con ciò si pensava di poter aprire la strada ad un mutamento nella valutazione morale della contraccezione.

Di questo avviso erano state le riflessioni di Herbert Doms (Doms 1935). La dottrina tradizionale sulla gerarchia dei fini era stata però ripresa da Pio XI nell'enciclica Casti Connubii (Pio XI, Enc. Castii Connubii, AAS 22 [1930], 546) e ribadita in una risposta del Sant'Uffizio, che respingeva l'opinione contraria (Decr. S. Officii, 29 Mart. [1 Apr.] 1944: AAS 36 [1944], 103: DH 3838). Ora il fatto che Gaudium et spes aveva preferito non servirsi del linguaggio dei fini fu interpretato come segno di un cambiamento della dottrina stessa, preludio ad una corrispondente revisione della proibizione etica. In realtà la scelta di evitare questo linguaggio era stata esplicitamente motivata nelle discussioni in aula come privilegio per un linguaggio pastorale e non come mutamento della dottrina (Cf. Turbanti 2000, 358-368). E proprio con questa precisazione era stata approvata dai Padri.

La costituzione conciliare arrivò ad un punto di equilibrio valorizzando sia l'amore coniugale (GS 49) sia la procreazione (GS 50), e segnalandone l'intima connessione (GS 51). E tuttavia il testo riflette in qualche modo alcune carenze dovute al contesto culturale dell'epoca, che era segnato da un forte allarme, lanciato dal Club di Roma, che portava a sentire solo la preoccupazione di controllo demografico; da un'incapacità di cogliere il valore positivo della natura per l'amore ${ }^{15}$; e da un'accentuata privatizzazione della famiglia, che ne smarriva l'importanza per il bene comune della società e non solo per gli individui (Cf. Granados 2013, 82-83. Al riguardo si veda anche: Bordeyne 2010).

L'interpretazione personalista di Doms, così come quella che legge Gaudium et spes in discontinuità rispetto alla dottrina classica, riflette una duplice limitazione, sia nell'intendere il valore della procreazione, sia nel concepire il primato dell'amore all'interno della vita coniugale.

In effetti il fine procreativo viene inteso come fine meramente biologico, quando non addirittura fisiologico, della relazione sessuale. Dunque viene collocato ad un livello infra-personale e infra-umano. Il dualismo antropologico e la svalutazione della corporeità diventano fattore di disprezzo della procreazione, vista con sospetto e con precauzione.

\footnotetext{
${ }^{15}$ Interessante l'analisi di Lear 1991, che evidenzia le risorse della psicoanalisi freudiana contro la deriva nichilistica nell'interpretazione dell'amore al di fuori della natura, che è poi maturata nella Gender Theory.
} 
D'altro canto l'affermazione del primato dell'amore nella gerarchia dei fini, si basa sull'equivoco di identificare l'aiuto reciproco con l'amore. In realtà l'amore non è un fine particolare tra gli altri, ma la realtà spirituale, che coordina e armonizza il raggiungimento dei diversi fini. Lo esprime bene San Giovanni Paolo II in una delle ultime Catechesi del mercoledì, nelle quali spiega anche la concordia tra Gaudium et spes e Humanae vitae su questo punto della gerarchia dei fini. Vale la pena rimeditare queste parole:

Secondo il linguaggio tradizionale, l'amore, quale "forza" superiore, coordina le azioni delle persone, del marito e della moglie, nell'ambito dei fini del matrimonio [...]. L'amore, come forza superiore che l'uomo e la donna ricevono da Dio insieme alla particolare "consacrazione" del sacramento del matrimonio, comporta una coordinazione corretta dei fini [...]. La dottrina della costituzione Gaudium et spes, come pure quella dell'enciclica Humanae vitae, chiariscono lo stesso ordine morale nel riferimento all'amore, inteso come forza superiore che conferisce adeguato contenuto e valore agli atti coniugali secondo la verità dei due significati, quello unitivo e quello procreativo, nel rispetto della loro inscindibilità. In questa rinnovata impostazione, il tradizionale insegnamento sui fini del matrimonio (e sulla loro gerarchia) viene confermato e insieme approfondito... (Giovanni Paolo II 2009, cat. 123 [10 ottobre 1984], 479).

Come si vede, staccare l'amore dalla concretezza dei beni del matrimonio, che dovrebbe promuovere, e farne invece un bene tra gli altri, significa averne una concezione astratta ed individualista. Staccato dalla natura, l'amore diventa un'emozione, mentre se si sgancia dal riferimento ai beni, si privatizza. E invece, "amare è volere il bene della persona", come afferma in modo semplice e icastico san Tommaso d'Aquino(San Tommaso d'Aquino, Summa contra Gentiles, 1. III, c. 90 [n. 2657]). I beni sono dunque la mediazione necessaria dell'amore tra le persone, che permettono e specificano la costruzione di una comunione nella trama della società e nel cammino di una storia.

\section{Quale coscienza?}

Al centro delle indicazioni "pastorali" sull'enciclica Humanae vitae, rivolte ai fedeli dalle Conferenze episcopali, si colloca il tema della coscienza, che viene intesa come mediazione necessaria del soggetto rispetto alla generalità dei principi. Ed è proprio basandosi sull'insegnamento di Gaudium et spes che sono state proposte soluzioni volte a giustificare eccezioni rispetto alla norma morale. Così la Conferenza episcopale tedesca difese la legittimità di seguire un'opinione diversa rispetto a quella proposta dal Papa nel suo magistero ordi- 
nario, qualora le argomentazioni non risultassero soggettivamente convincen$\mathrm{ti}^{16}$. E i Vescovi francesi sostennero la possibilità della coscienza di eccepire alla norma in caso di "conflitto di doveri". Vale la pena di saggiare che concezione di coscienza sia sottesa a queste indicazioni, in riferimento al documento conciliare e all'enciclica stessa di Paolo VI.

Il breve paragrafo 16 di Gaudium et spes, contiene una grande densità di elementi e di dottrina, non sempre perfettamente amalgamati tra loro, così da meritare la qualifica di "testo di compromesso" (Kompromißtext; Cf. Anaya 1993). E tuttavia un'ermeneutica corretta non può appellarsi allo "spirito" del Concilio, per mettere da parte la "lettera" effettivamente approvata dai Padri. In effetti, se la coscienza è definita come «il nucleo più segreto e il sacrario dell'uomo, dove egli si trova solo con Dio, la cui voce risuona nell'intimità propria», il testo menziona anche che è «nell'intimo della coscienza che l'uomo scopre una legge che non è lui a darsi, ma alla quale deve obbedire», nello sforzo di «conformarsi alle norme oggettive della moralità».

Da parte sua, Humanae vitae, parla della coscienza dei coniugi nel contesto alla loro vocazione alla "paternità responsabile" (n. 10) e afferma che essa, quando è retta, è anche «fedele interprete dell'ordine morale oggettivo stabilito da Dio». La metafora dell'interprete implica da parte della coscienza la capacità di parlare due lingue: da una parte quella della norma e dall'altra quella delle circostanze della vita, in ordine a stabilire quale sia la volontà di Dio nel concreto. Il richiamo alla necessaria rettitudine suggerisce che la trasparenza alla verità è assicurata non semplicemente da un'autenticità soggettiva, ma dalla cura delle virtù morali. Così è possibile superare l'orizzonte ristretto della contrapposizione tra norma e coscienza, nell'orizzonte più tomista della prudenza, che colloca il momento del giudizio all'interno della prospettiva del soggetto e del dinamismo dell'agire (Cf. S. Pinckaers 1996, 126-141; Melina 2018).

La coscienza quindi non è arbitra del bene e del male, che deve invece cercare di scoprire a partire dai principi oggettivi e dagli insegnamenti della Chiesa. Non è neppure una mera esecutrice di precetti, perché deve invece scoprire una chiamata al bene singolare, determinata dalle circostanze. In questo senso la responsabilità procreativa è compito altissimo e peculiare dei coniugi in collaborazione con Dio (pro-creazione). E d'altra parte i coniugi cristiani non possono ritenere leciti, in nome della loro coscienza, dei metodi di contraccezione che il Magistero della Chiesa ha definiti intrinsecamente cattivi. Infatti, accanto alla funzione interpretativa della coscienza sul piano singolare, Paolo

${ }^{16}$ È la famosa Königsteiner Erklärung del 30 agosto 1968, finora confermata dai Vescovi tedeschi. Nello stesso senso le dichiarazioni dei Vescovi austriaci e belgi. Cf. Tettamanzi 1969. 
VI definisce anche il compito interpretativo del Magistero della Chiesa nei confronti della legge naturale (n. 4) a livello universale.

Si può così misurare quanto contrasti con la dottrina cattolica sulla coscienza, quella che è stata anche recentemente proposta quale "cambiamento di paradigma" sulla scorta di Amoris laetitia, ad esempio dal teologo tedesco Peter Hünermann, il quale pensa che, dal momento che l'universalità dei principi e dei comandamenti non può mai raggiungere la singolarità e la particolarità delle situazioni individuali, va sempre attribuita alla coscienza l'insostituibile libertà di decidere, anche in contrasto con quanto stabilito da una norma morale negativa, che proibisce azioni in se stesse intrinsecamente cattive (Cf. il suo intervento nell'opera collettiva: Th. Knieps-Port le Roi [ed.] 2017). Per questo, secondo il teologo di Tübingen, questa nuova visione della coscienza esigerebbe la correzione di certi passaggi di Humanae vitae, di Familiaris consortio, del Catechismo della Chiesa Cattolica e di Veritatis splendor, «perché contengono un' (indebita) estensione della legge etica naturale nella particolarità delle azioni individuali». Sulla questione dell'interpretazione del valore normativo dell'insegnamento di Humanae vitae, si gioca dunque non solo un precetto di vita coniugale, ma una visione stessa della morale, così come è stata insegnata finora dalla tradizione della Chiesa e in particolare dal Beato Paolo VI, da San Giovanni Paolo II e da Benedetto XVI.

\section{Quale pastorale?}

Un ultimo, decisivo punto del confronto tra Gaudium et spes e Humanae vitae, che vorremmo, sia pur brevemente, suggerire, è quello che riguarda la qualità pastorale dell'insegnamento, che viene impartito nei due documenti. Non si vuole con ciò alludere al carattere "non definitorio", e quindi solo pratico, cui taluni commentatori hanno voluto ridurre l'autorevolezza del pronunciamento di papa Paolo VI, allo stesso modo in cui talvolta si vuole ridimensionare il valore dottrinale del Concilio Vaticano II (È questa la tesi di Mongillo, Chiavacci, Goffi 1968). Si intende piuttosto qualificare uno specifico e nuovo registro di esercizio del magistero, iniziato con la costituzione conciliare e che si porrebbe come chiave ermeneutica della stessa enciclica sulla regolazione della natalità. In altre parole, Gaudium et spes inaugurerebbe una modalità di insegnamento preoccupata soprattutto del dialogo col mondo contemporaneo, che dovrebbe permettere una rilettura "non formale, non moralistica e non anti-moderna" dell'insegnamento di Humanae vitae (È la tesi di Marengo 2014, 161-172). Tale conversione pastorale sarebbe richiesta ora anche da papa Francesco, come stile necessario di una Chiesa "in uscita missionaria" (Cf. Francesco, Es. ap. Evangelii gaudium, 25-26). 
In questa lettura, al termine "pastorale" viene attribuita la valenza di una originalità pratica, in opposizione dialettica rispetto all'elemento dottrinale. Lungi dal rivestire un carattere solo applicativo, il tratto pastorale verrebbe a godere di una sua autonomia e addirittura di un primato sulla dottrina, così da riflettere una concezione di praxis molto simile a quella propria dell'ideologia marxista (Cf. Pérez-Soba 2014, 71-102). Mentre la dottrina, preoccupata dell'ortodossia, comporterebbe irrigidimenti e costringerebbe all'immutabilità, il primato dell'ortoprassi assicurerebbe un'idea storica ed evolutiva della verità, con la duttilità di adattarsi alle circostanze storiche e alle situazioni personali, "caso per caso". Si tratta, in qualche modo, di una concezione modernista, se non hegeliana, di verità, che si fa nella storia. Gli insegnamenti precedenti potrebbero essere accolti solo liberandoli dal fissismo della comprensione letterale e permettendo allo Spirito di far progredire il popolo in un'esperienza sempre nuova (Cf. Fontana 2018). Si tratterebbe perciò di adattare al mondo il linguaggio della Chiesa e specialmente la sua morale.

In realtà non è questo il senso della pastoralità inteso da Gaudium et spes. Nella famosa prima nota apposta al titolo, che spiega la qualifica "pastorale" della costituzione, si parla di atteggiamento di dialogo col mondo sulla base di "principi dottrinali" "immutabili", che determinano una visione dell'uomo e del mondo, a partire dalla quale poi si possono illuminare questioni che implicano elementi più "contingenti", legati a "circostanze mutevoli". Nello stesso senso l'enciclica Humanae vitae, rivolgendosi ai sacerdoti li invitava al loro «primo compito, che è quello di «esporre senza ambiguità l'insegnamento della Chiesa sul matrimonio» (n. 28), poiché «non sminuire in nulla la salutare dottrina di Cristo è eminente forma di carità verso le anime» (n. 29).

La dottrina non va quindi contrapposta alla pastorale, poiché essa è "il corpo (vivente) della verità", secondo l'incisiva espressione di Sant'Ireneo di Lione $^{17}$. Essa getta una luce, che orienta l'azione della Chiesa e genera una pienezza di vita. Per questo il tratto innovativo del Vaticano II, ripreso in particolare da papa Francesco, non può essere inteso come una rivoluzione, che mette da parte la preoccupazione per la verità, o che relega quest'ultima nel museo dei reperti storici ormai obsoleti.

Una chiave feconda di lettura dell'intenzione pastorale del recente Concilio venne piuttosto offerta dall'Arcivescovo di Cracovia, il card. Wojtyła, con l'idea di "arricchimento della fede" (Wojtyła 1981). Pur non formulando nuovi dogmi, il magistero del Concilio ha inteso aiutare i fedeli ad un più intimo e profondo radicarsi della fede nella vita e così ha avuto un influsso anche dot-

${ }^{17}$ Sant'Ireneo di Lione, Adversus haereses, II, 25, 2, citato in Granados 2015. In questo saggio, dedicato all'intima unità tra dottrina e pastorale, si veda soprattutto il cap. V: "La dottrina, luce pastorale", 111-127. 
trinale. Il profilo pastorale è dunque da scorgere nell'intenzione di arricchire la fede promuovendo una rinnovata esperienza della sua capacità di accogliere e dilatare dall'interno l'humanum. In tal senso l'esperienza dell'amore, e in modo specifico quella della famiglia, è stato visto dai Padri, nella Gaudium et spes, come il luogo strategico per un rinnovato dialogo della Chiesa col mondo (In merito: Pérez-Soba [a cura di] 2016).

\section{Conclusione}

Che cosa è in gioco con l'Humanae vitae, o meglio con le riletture che ne vengono proposte, in nome di un "cambiamento di paradigma" per la morale cattolica? A prima vista sembra che si tratti solo di un rinnovamento pastorale nel senso di una flessibilità misericordiosa, che riconosce il primato della persona e della coscienza. Il confronto con la costituzione pastorale conciliare Gaudium et spes e con le sue molteplici interpretazioni, ci ha permesso di verificare che è in gioco qualcosa di più sostanziale: l'antropologia cristiana, la dottrina del sacramento del matrimonio, la visione sui fondamenti della morale e in particolare l'idea di coscienza, e finalmente l'autenticità dell'azione pastorale della Chiesa come azione salvifica, che prosegue quella del Buon Pastore.

La recente lettera della Congregazione per la Dottrina della Fede Placuit Deo su alcuni aspetti della salvezza cristiana, approvata da Papa Francesco il 16 febbraio scorso, ci offre un nuovo punto di vista sintetico, alla luce del quale considerare il cammino di riflessione percorso. Essa ammonisce sul perenne riproporsi anche ai nostri giorni di due antiche eresie: quella pelagiana, che pensa alla salvezza come ad un'autorealizzazione individualistica basata sulle capacità umane, e quella gnostica, che la riduce ad un'esperienza puramente interiore, senza assumere la concretezza del nostro corpo e delle relazioni che ci legano agli altri e al mondo creato.

Non è forse proprio questo che abbiamo visto riproporsi anche nelle interpretazioni di Gaudium et spes e nelle corrispondenti riletture di Humanae vitae? Quando si scardina l'amore dalla carne, quando si stacca la persona dalla natura e quando si separa il matrimonio dalla procreazione si consegna la persona all'arbitrio e all'illusione di una libertà fittizia, di un amore astratto. "La carne è il cardine della salvezza", dicevano i Padri (Tertulliano, De resurrectione mortuorum VIII, 6-7: «Caro cardo est salutis». Cf. Granados Siena 2010). L'uomo si salva solo se salva la propria carne. Per questo è così importante la norma morale insegnata da Humanae vitae sulla contraccezione: essa custodisce l'integralità e la verità del dono coniugale. Essa è anche la "pietra di paragone" dell'antropologia cristiana di Gaudium et spes. 
Sommario

Il conflitto delle interpretazioni sull'enciclica Humanae vitae di Papa Paolo VI nel suo rapporto con la costituzione pastorale Gaudium et spes del Concilio Vaticano II, si è riacceso con l'innesco dell'idea che Amoris laetitia rappresenti un "cambio di paradigma" per la pastorale. Il saggio esplora i contenuti del dibattito teologico intorno a quattro tematiche: l'antropologia (quale personalismo?), la teologia del matrimonio (quale amore?), la morale (quale coscienza?), la prassi ecclesiale (quale pastorale?).

$\mathrm{Ne}$ risulta che nell'interpretazione di Humanae vitae è in gioco ultimamente la verità dell'incarnazione contro una deriva neo-gnostica, che scardina l'amore dalla carne, la persona dalla natura, il matrimonio dalla procreazione e che finisce per abbandonare la dottrina della Chiesa alle convenienze di un ambiguo primato della pastorale.

Parole chiave: personalismo; naturalismo; cambio di paradigma; teologia del corpo; beni del matrimonio; paternità responsabile; pastorale coniugale; neo-gnosticismo

\section{INTERPRETATIONS OF GAUDIUM ET SPES AND RECEPTION OF HUMANAE VITAE}

\section{Summary}

The conflict of interpretations on Pope Paul VI's encyclical Humanae vitae in his relationship with the pastoral constitution Gaudium et spes of the Vatican Council II was rekindled with the triggering of the idea that Amoris laetitia represents a "paradigm shift" for the pastoral. The essay explores the contents of the theological debate around four themes: anthropology (which personalism?), the theology of marriage (what love?), morality (what conscience?), ecclesial practice (which pastoral care?).

It results that there is the truth of the incarnation against a neo-gnostic drift in the interpretation of Humanae vitae, which unhinges love from the body, the person from nature, marriage from procreation and which ends up abandoning the doctrine of Church for the conveniences of an ambiguous primacy of pastoral care.

Keywords: personalism; naturalism; „paradigm shift”; theology of the body; the goods of marriage; responsible parenthood; spouses' pastoral care; neognosticism

Słowa kluczowe: personalizm; naturalizm; „zmiana paradygmatu”; teologia ciała; dobra małżeństwa; odpowiedzialne rodzicielstwo; duszpasterstwo małżonków; neognostycyzm 


\section{BIBLIOGRAFIA}

Anaya L.A. (1993), La conciencia moral en el marco antropológico de la constitución pastoral Gaudium et spes, Buenos Aires.

Bordeyne Ph. (2010), Éthique du mariage. La vocation sociale de l'amour, Paris.

Commissione Teologica Internazionale (2009), Alla ricerca di un'etica universale: nuovo sguardo sulla legge naturale, Città del Vaticano.

Delhaye Ph. (1968), L'enciclique "Humanae vitae" et l'ensiegnement de Vatican II sur le mariage et la famille (GS), w: Bijtragen. Tijdschrift voor filosophie en theologie 29, s. 351-368.

Doms H. (1935), Vom Sinn und Zweck der Ehe, Breslau.

Fernández Benito A. (1994), Contracepción: de Vaticano II a la Humanae vitae, Toledo.

Fontana S. (2018), Addio insegnamento, ora il magistero pone dubbi, w: La nuova Bussola quotidiana, http://www.lanuovabq.it/it/addio-insegnamento-il-magistero-ora-pone-dubbi [dostęp: 03.04.2018].

Fuchs J. (1996), Ricercando la verità morale, Cinisello Balsamo.

Gałuszka P.S. (2017), Karol Wojtyła e „Humanae vitae”. Il contributo dell'Arcivescovo di Cracovia e del gruppo dei teologi polacchi all'enciclica di Paolo VI, Siena.

Gil Hellin F. (1998), Intervención conciliar de Pablo VI sobre el matrimonio y la familia, „Anthropotes" XIV/2, s. 359-372.

Giovanni Paolo II (2009), L'amore umano nel piano divino. La redenzione del corpo e la sacramentalità del matrimonio nelle catechesi del mercoledi (1979-1984), a cura di G. Marengo, Città del Vaticano.

Goertz S., Witting C. red. (2016), Amoris laetitia. Wendepunkt für die Moraltheologie?, Freiburg im Br., s. 13-79.

Granados J. (2010), La carne si fa amore. Il corpo, cardine della storia della salvezza, Cantagalli, Siena 2010.

Granados J. (2013), Il Memoriale di Cracovia. Introduzione, w: Persona e natura. Persona e natura nell'agire morale, red. J.J. Pérez-Soba, P. Gałuszka, Siena, s. 81-86.

Granados J. (2014), Una sola carne in un solo Spirito. Teologia del matrimonio, Siena.

Granados J. (2015), Eucaristia e divorzio: cambia la dottrina?, Siena.

Häring B. (1971), Morality is for Persons. The Ethics of Christian Personalism, New York.

Janssens L. (1972), Ontic Evil and Moral Evil, „Louvain Studies” 4/2, s. 115-156.

Kasper W. (2016), „Amoris laetitia”: Bruch oder Aufbruch. Eine Nachlese, „Stimmen der Zeit” 11, s. $723-732$.

Knieps-Port le Roi Th. red. (2017), A Point of No Return? "Amoris laetitia” on Marriage, Divorce and Remarriage, Intams Studies on Marriage \& Family nr 2, Münster.

Lear J. (1991), Love and its Place in Nature. A Philosophical Interpretation of Ferudian Psychonalysis, New York.

Marengo G. (2014), Generare nell'amore. La missione dellla famiglia cristiana nell'insegnamento ecclesiale dal Vaticano II ad oggi, Assisi.

Maritain J. (1985), Nove lezioni sulla legge naturale, red. F. Viola, Milano.

Mattheeuws A. (1989), Union et procréation. Developpements de la doctrine des fins du mariage, Paris.

Mazzocato G. (2006), Il dibattito tra Doms e neotomisti sull'indirizzo personalista, „Teologia” 31, s. $249-275$.

Melina L., Pérez-Soba J.J. (red.) (2012), La soggettività morale del corpo (VS 48), Siena.

Melina L. (1987), La conoscenza morale. Linee di riflessione sul Commento di san Tommaso all'Etica Nicomachea, Roma.

Melina L. (2006), La prudenza nella vita morale secondo S. Tommaso, „Aquinas” 49/2, s. 381-393.

Melina L. (2018), Coscienza e prudenza. La ricostruzione del soggetto morale cristiano, Siena.

Mongillo D., Chiavacci E., Goffi T. (1968), „Humanae vitae”. Note teologico-pastorali, Brescia. 
Pérez-Soba J.J. (2011), La verità dell'amore: una luce per camminare. Esperienza, metafisica e fondamento della morale, Siena.

Pérez-Soba J.J. (2013), La natura della persona si rivela nelle sue azioni. Status quaestionis, w: Persona e natura nell'agire morale, red. J.J. Pérez-Soba, P. Gałuszka, Cantagalli, Siena, s. 33-78.

Pérez-Soba J.J. (2014), Verità dottrinale e verità pastorale, w: Misericordia, verità pastorale, red. J.J. Pérez-Soba, Siena, s. 71-102.

Pérez-Soba J.J., Gałuszka P. red. (2013), Persona e natura nell'agire morale, Siena.

Pérez-Soba J.J. red. (2016), La famiglia: chiave del dialogo Chiesa-Mondo nel $50^{\circ}$ della „Gaudium et spes", Siena.

Pinckaers S. (1996), Coscienza, verità e prudenza, w: Aa.Vv. (a cura di G. Borgonovo), La coscien$z a$, (Conf. Intern. "Wethersfield Institute" - Orvieto: 27-28 maggio 1994), Città del Vaticano, s. $126-141$.

Pius XI (1930), Encyklika Castii connubii, „Acta Apostolicae Sedis” 22, s. 539-592.

Ratzinger J. (1966), Theological Highlights of Vatican II, New York-Mahwah.

Rigobello A. (1975), Il personalismo, Roma.

Schockenhoff E. (2017), Traditionsbruch oder notwendige Weiterbildung? Zwei Lesearten des Nachsynodalen Schreibens ,Amoris laetitia”, „Stimmen der Zeit” 3, s. 147-158.

Tettamanzi D. (1969), La risposta dei vescovi alla „Humanae vitae”. I documenti degli episcopati nazionali. Commento e direttive pastorali, Milano.

Turbanti G. (2000), Un Concilio per il mondo moderno. La redazione della costituzione pastorale „, Gaudium et spes” del Vaticano II, Bologna.

Wojtyła K. (1973-1974), Wypowiedź wstępna w czasie dyskusji nad „Osoba i czynem” w Katolickim Uniwersytecie Lubelskim, dnia 16 XII 1970 r., „Analecta Cracoviensia” 5-6, s. 53-55, Stowo końcowe, s. 243-263.

Wojtyła K. (1978), La visione antropologica della „Humanae vitae”, „Lateranum” XLIV, s. 125-145 .

Wojtyła K. (1981), Alle fonti del rinnovamento. Studio sull'attuazione del Concilio Vaticano Secondo, Città del Vaticano.

Livio Melina - ks. prof., od 1980 roku kapłan diecezji Adria, teolog włoski, profesor teologii moralnej w Papieskim Instytucie Jana Pawła II dla Nauk o Małżeństwie i Rodzinie w Rzymie, którego rektorem był w latach 2006-2016. Visiting Professor w Waszyngtonie (DC) w Stanach Zjednoczonych oraz w Melbourne w Australii, od 2007 roku członek zwyczajny Papieskiej Akademii Teologicznej. W 2013 roku otrzymał doktorat honoris causa Uniwersytetu Anáhuac w Meksyku. Jest redaktorem naukowym czasopisma „Anthropotes”, członkiem komitetu redakcyjnego francuskiego czasopisma „Revue Théologique des Bernardins” oraz komitetu włoskiego konsultorów międzynarodowego czasopisma teologicznego „Communio”. Współpracuje także z czasopismami: „The Tomist”, „La Scuola Cattolica”, „Medicina e Morale”, „La Società”, „Il Nuovo Areopago”, „Logos”, „Path”. Członek paryskiej Académie d'Education et d'Etudes Sociales. Autor licznych książek i publikacji z zakresu teologii moralnej fundamentalnej, etyki seksualnej i małżeńskiej oraz bioetyki. 